\title{
A Review of Data Fusion Techniques
}

\author{
Afnan Alofi \\ Department of Electrical and \\ Computer Engineering, FOE, \\ King Abdulaziz University, \\ Jeddah, KSA
}

\author{
Anwaar Alghamdi \\ Department of Electrical and \\ Computer Engineering, FOE, \\ King Abdulaziz University, \\ Jeddah, KSA
}

\author{
Razan Alahmadi \\ Department of Electrical and \\ Computer Engineering, FOE, \\ King Abdulaziz University, \\ Jeddah, KSA
}

\author{
Najla Aljuaid \\ Department of Electrical and Computer \\ Engineering, FOE, \\ King Abdulaziz University, \\ Jeddah, KSA
}

\author{
Hemalatha $\mathrm{M}$. \\ Department of Electrical and Computer \\ Engineering, FOE, \\ King Abdulaziz University, \\ Jeddah, KSA
}

\begin{abstract}
In many cases, researchers use more than one sensor and synthesize their raw data to generate more meaningful information that can be of greater value than single source data. The process of merging multiple data and knowledge from different sources to represent the object into a regular, accurate, useful, meaningful representation is known as data fusion. This article summarizes the state of data fusion and compares relevant techniques. We explain possible data fusion classifications and review the most common fusion methods such as Kalman filter and The Bayesian Methods. Then we evaluate these methods and discuss the advantages and disadvantages of each method.
\end{abstract}

\section{General Terms}

Multi-sensor fusion, data fusion, Kalman filter, Particle filter, Bayesian methods, Dempster-Shafer.

\section{Keywords}

Fusion, sensor, filter.

\section{INTRODUCTION}

The combination of data from multiple sensors called data fusion. These sensors may have same or different type also the same or different location. The accuracies of the data fusion is better than the accuracies could found by the single sensor.

The first architecture of data fusion technique was proposed in the 1980s [1]. Then, the data fusion was implemented on the robotics field on the United States of Americas. In 1988 a public data structure based on the data fusion technique was be offered. Also, the hierarchical architecture was presented in the same year [2].

There are several model was presented for the data fusion which are the Durrant-Whyte data fusion model that could use in the robotics field and it was obtained in 1988. In 1993 there is another model was developed which is the data fusion and resources management model. The decision-sensor data fusion was displayed in the LASS architecture in 1998. By now data fusion have to use in more application such as the military applications. Also, the nonmilitary applications that is include monitoring of manufacturing processes. For example, the manufacturing could use the data fusion to control and determine the factory. Also, $\mathrm{t}$ can be used in environmental and health care [2].
There are several advantages of multisensory system which can be summarized as the following; increasing the reliability when sensor fails, increasing the estimation confidence and decrease the uncertainty of it, expanding discrimination ability due to more comprehensive information arriving from multiple sensors and finally enhancing the resolution due to usage of multiple sensors. However, there are also some difficulties in implementing the sensor fusion such as the divers format of the data and the dimensionality of the data.

\section{DIFFERENT CLASSIFICATION OF DATA FUSION TECHNIQUES}

Data fusion could partition into various classifications in view of some criteria. The sections below show some of the possible classifications of data fusion.

\subsection{Sensor Configuration}

The data fusion could be categorized based on the sensor configuration as follows:

\section{Complementary:}

The complementary configuration is a combined of sensors in order to give a complete image of the phenomenon under observation since each sensor could observe different part of the same environment.

This type of fusion is the easiest type since the data appended to each other from independent sensors. The most example of the complementary fusion is the employment multiple numbers of cameras each observing disjunction of the room's parts.

\section{Competitive:}

The competitive sensors are a collection of independent data of the same property.

\section{Cooperative:}

The information provided by two or more independents sensor is called cooperative sensors where sometime the single sensor would not provide that information.

The main disadvantage of the cooperative sensor that it may decrease the accuracy of the data [3].

\subsection{Input/Output Configuration}

The input-output categories were be classified based on the three abstraction levels which are raw data level, feature level and decision level where the processed data comes from the raw data level and the results belong to the feature level. 
Dasarathy has arranged the data fusion into forms got from the deliberation level of the procedures information and output information.

The explanation behind the Dasarathy model was the presence of combination ideal models where the information and yield of the data fusion procedure have a place with different levels. Cases are highlight choice and extraction, since the prepared information comes from the crude information level and the outcomes have a place with the element level. These uncertain combination standards in some cases have been appointed as per the level of their information and in some cases as indicated by the level of their yield information [4].

To keep away from these order issues, Dasarathy expanded the three-level view to the following five classes:

\section{1. data in-data out (DAI-DAO):}

Data in - data out is the most fundamental or basic data fusion technique. It is processing input and output raw data to get accurate outcomes where data fusion at this level is directed promptly after the information are accumulated from the sensors.

\section{Data in-feature out (DAI-FEO):}

This level using the raw data to extract features and characteristics describing the environment of the object.

\section{Feature in-feature out (FEI-FEO):}

This level has to featuring the data fusion to obtain new features for it so all of input and output will be featured.

\section{Feature in-decision out (FEI-DEO):}

This level could obtain a set of features as input and provides a set of decisions as output.

\section{Decision In-Decision Out (DEI-DEO)}

This level has to merge the input decision to obtain a new decision output.

\subsection{Classification based on the type of architecture}

There are several architecture types using in designing data fusion. The following will present those types [5]:

\section{Centralized architecture:}

The information from all the input sources will be received by the fusion node in the central processor where the informational was received by the fusion node is an observational measurement. Then the fusion process will obtain in the central process.

There are some disadvantages of a centralized architecture that refer to the amount of bandwidth requires sending the raw data. Also, the delays occur accord to the data transferring will affect the results of data.

\section{Decentralized architecture:}

The decentralized architecture is consisting of numbers of nodes where each node has a specific processor and the data fusion not occurring in a specific node. So, each node fuses its current data with the data received from its peer.

The increasing numbers of nodes cause an increment in the communication cost and that is one of the disadvantages of decentralized architecture.

\section{Distributed architecture:}

In this architecture each input source have to process the measurements before transmitted to the fusion node that's mean the completing of the state estimation and the data association are done in source node before communicate it to the fusion node. Then, the estimation state for the object will provide by each node depends on the local view of that object.

This type of architecture provides different options and variations that range from only one fusion node to several intermediate fusion nodes.

\section{Hierarchical architecture:}

The hierarchical architectures is a set of the above two architectures which are decentralized and distributed architectures. Where this two architectures are difficult to implement it singly. However, the requirements, demand, data availability and organization of data fusion system will be responsible for selecting the best architecture.

As shown previously the data fusion could be categorized based on several criteria. This paper will presents the data fusion methods classified into Estimation and Decision methods.

\section{STATE ESTIMATE METHODS}

State estimation is a common phase in data fusion algorithms. The state estimation methods are used to determine the state of a desired system that is continuously changing given some observations or measurements. In the context of data fusion, the state that is being estimated is denoted by $\mathrm{x}$, and $\mathrm{F}$ represents the sequence $\mathrm{k}$ of the previous observations of $\mathrm{x}$. Two of the most known and used estimation methods are the Kalman filter (KF) and the Particle filter (PF). They are presented in section 3.1 and 3.2, respectively.

\subsection{Kalman Filter}

Kalman filtering provides a recursive method of estimating the dynamical system state with the presence of uncertainty. It is one of the most useful and common estimation techniques where it is easy to implement on linear systems. For nonlinear systems, it is best to implement the variant version of Kalman filtering; called the Extended Kalman Filter (EKF).

The equations below [(1) and (2)] can be implemented directly or even by modifying them to enhance the accuracy of the estimation when there is a necessity to address nonGaussian noise model, for example, or map problems or even to reduce computational complexity [6]. The Kalman filter equations are as follow:

$\hat{x}_{k}=F_{k} \hat{x}_{k-1}+B_{k} \vec{u}_{k}$

$P_{k}=F_{k} P_{k-1} F_{k}^{T}+Q_{k}$

The vector $\hat{x}_{k}$ is the estimate state of the full system state $x_{k}$. $P_{k}$ is the predicted covariance matrix of the error. These two equations provide a vector estimate and an estimate of the error covariance associated with it. Where $F$ is a matrix that represents the dynamics of the system where it helps to look at the current state and predict the next state at time $k . B$ is the control matrix that describes how inputs control the dynamics. The matrix $Q$ is the noise covariance. The noise here meant to consider unmodeled disturbances that affect system dynamics, e. g., wind or slipping wheels [6][7].

The above equations are used to generate new estimate from previous estimate plus a correction for known external influences $(u)$, along with its new uncertainty which is predicted from the old uncertainty with additional external 
uncertainty $(Q)$. This estimate is not necessarily accurate. The Kalman filter involves another stage to update these estimates. The update equations are given by:

$$
\begin{aligned}
& \hat{x}^{\prime}{ }_{k}=\hat{x}_{k}+K^{\prime}\left(\vec{z}_{k}-H_{k} \hat{x}_{k}\right) \\
& P^{\prime}{ }_{k}=P_{k}-K^{\prime} H_{k} P_{k}
\end{aligned}
$$

Where:

$$
K^{\prime}=P_{k} H_{k}^{T}\left(H_{k} P_{k} H_{k}^{T}+R_{k}\right)^{-1}
$$

In the above equations: $z$ is the measurements vector, it usually comes from the sensors readings. $H$ is the transformation matrix that is used to map state vector parameters into the measurement domain. And $\mathrm{R}$ is the covariance matrix of the measurements noise [8].

The Kalman gain $(\mathrm{K})$ describes the amount of update needed at each recursive estimation. $\mathrm{K}$ can be viewed as the weighting factor that considers the relationship between the accuracy of the predicted estimate and the measurement noise. High values of $\mathrm{K}$ mean that the estimate is making bigger updates based on the input measurements, and that measurements contain smaller noise.

The above equations provide the best estimate of state of the system $x$. These equations minimize the error between $x_{k}$ and $\hat{x}_{k}$ at every time $k$

\subsection{Particle Filter}

Particle filtering is a recursive implementation of the sequential Monte Carlo method [9]. The particle filter allows analysis of complex systems, it provides better results for nonlinear systems with a non-Gaussian noise. It is used to approximate the posterior estimation by a set of random state samples drawn from this posterior. These samples are called particles and are denoted by the set:

$$
X_{k}:=\left\langle x_{k}^{1}, w_{k}^{1}\right\rangle,\left\langle x_{k}^{2}, w_{k}^{2}\right\rangle, \ldots,\left\langle x_{k}^{N}, w_{k}^{N}\right\rangle
$$

Where each $x_{k}^{[n]}$ represents a hypothesis of the realworld state at time $k$ with its importance weight $w_{k}^{[n]}$. The number of particles $(N)$ is usually a substantial number, e. g., $N>1,000$. These particles are increased over time with a sampling and resampling phases [10]. Particle filters generally comprise the following steps:

\section{Prediction}

For each particle, the state of the system is predicted at time $\mathrm{k}$ with distribution noise $(q)$, as in equation [6]

$\widehat{X}_{k}^{n}=F_{k-1} \widehat{X}_{k-1}^{n}+q_{k}$

Where $\mathrm{F}$ is the transition matrix of the system.

\section{Particles weight update}

The predicted observation state of the system (z) is used to evaluate the likelihood of each particle.

$$
\hat{z}_{k}^{n}=H_{k} \widehat{X}_{k}^{n}+r_{k}
$$

Where $H_{k}$ is the measurements matrix at time instant $\mathrm{k}$, and $r_{k}$ is the measurement noise. The unnormalized weight is denoted by:

$$
w^{n}=N\left(\hat{z}_{k}^{n}: z_{k}^{n}, \text { var }\right)
$$

And the normalized weight $\left(\widetilde{w}^{n}\right)$ :

$$
\widetilde{w}^{n}=\frac{w^{n}}{\sum_{i=1}^{N} w^{n}}
$$

\section{Resampling}

The cumulative weight $\left(W_{t}^{n}\right)$ denotes the number of times each particle is drawn on average. It is represented by the normalized weight times the number of particles. The cumulative weight is computed as follows:

$$
W_{t}^{n}=\sum_{i=1}^{n} \widetilde{w}^{n}
$$

This is used to generate uniform distributed random variables with number of steps equals to $\mathrm{N}$. Then each particle is decided whether to be removed or multiplied.

\section{Propagation}

The new values of the resampling at time $k-1$ are used to calculated the values at time $k$.

$$
\hat{x}_{k}^{1: N}=\hat{x}_{k-1}
$$

Then the posterior mean is computed:

$$
\hat{x}_{k}^{\prime}=\operatorname{mean}\left(\hat{x}_{k}^{n}\right), \quad n=1,2, \ldots, N
$$

These steps are repeated at each time instant $k$ [10]

\subsection{Evaluation of State Estimate Methods}

In robotics and intelligent systems tracking motions and state estimate is essential, however doing such thing faces a lot of problems and the most important one is external distortions and noises, which makes it difficult to absorb the data.

to overcome it, using filters seems ideal idea to filter out these noises and distortion to make accurate estimation. Whenever the system has liner inputted data such as position information, Kalman filters KF will be great solution, since it guarantee retrieving the optimal statistical estimation, and their simple mechanism they only needs the data from the previous state they don't need to keep large number of data to absorb, making them very fast to perform and suitable for real time problems and embedded systems, which are essential in tracking application [10].

However, not all the systems provides liner inputted data, for such thing some modification are needed to improve the KF, extended kalamen filter EKF are used for this purpose by linearizing those none liner data, unfortunately this linearization methods, made the EKF complex and making it difficult to implement and quit expensive, not to mentions that it may lead filter to be unstable, however EKF are popular in robotics, navigation application [11] and tracking GPS signals [12].

Moreover, more satiable and powerful approach for nonlinear estimation is Unscented Kalmen Filter UKF, it overcomes the pitfalls of the EKF by using sampling methods to finds the mean along with the covariance estimation, with minimal set number of sampling points, not to mention it grate feature of parallel implementation [10]. UKF is powerful in various of applications such as parameter estimation of time series modeling [13] and dynamic state estimation of power system network [14], however this is not the case in virtual reality VR applications, the quaternation motion estimation needed in VR showed that EKF was much appropriate from UKF since the quasi-liner of quaternation motion [12]. This concludes that 
there no good or bad type of KF, each one of them has it own strength and weaknesses, and each type will perform it best if it was used in it best application.

In the other hand, Particle Filter PF, is more flexible and accurate than KF if it used as multimodal estimator, since it ability to adopt nonlinear data, and its ability to show several estimations with single algorithm, it made more appropriate for multi-tracking systems [14]. However, contrariwise the $\mathrm{KF}$, the PF needs a large number of data inputs to gain small variance in estimates, such thing makes expensive in execution time [10][15].

\section{DECISION FUSION METHODS}

Decision is last step in fusion process. Decision can make by the knowledge that taken from previous steps and understated the situation. Also there are method help to make more accurate inference. In this section will present two most common methods which are Bayes method and Dempsrter Shafer theory method.

\subsection{The Bayes Method}

Bayes theory is probability theory and it use to describe or defined the probability of event that might happen under condition or anther event. The mathematically equation of Bayes theory is:

$$
P(A \mid B)=\frac{P(B \mid A) P(A)}{P(B)}
$$

Where:

- $\quad P(A)$ is the probability of event A just without any effect of any other event.

- $\quad$ P (B) is the probability of event B just without any effect of any other event.

- $\quad \mathrm{P}(\mathrm{B} \mid \mathrm{A})$ is the probability of event $\mathrm{B}$ given that $\mathrm{A}$ event is true.

In data fusion the Bayes theory use to provide meaning to data or evidence or information that collects or observed with prior beliefs about situation based on probability theory. The result of $\mathrm{P}(\mathrm{A} \mid \mathrm{B})$ condition probability will be in range between zero and one [10]. If the result was zero that means the event $\mathrm{P}(\mathrm{A}$ B) will never happen and if the result was one mean the event $\mathrm{P}(\mathrm{A} \mid \mathrm{B})$ will happen.

\subsection{Dempster Shafer Theory}

The Dempsrter Shafer theory is mathematical theory and it use to describes belief in hypothesis by define the maximum and minimum probability of hypothesis. The maximum probability of hypothesis $(\mathrm{H})$ is defined by belief function (bel):

$$
\operatorname{bel}(H)=\sum_{A \subseteq H} m(A)
$$

Where:

$\mathrm{m}(\mathrm{A})$ : The mass function or probabilities which satisfies $m(\varnothing)=0$.

The minimum probability of hypothesis $(\mathrm{H})$ is defined by plausibility function (pl):

$$
p l(H)=\sum_{A \cap H=\varnothing} m(A)
$$

The interval of confidence in hypothesis $(\mathrm{H})$ is $[\mathrm{bel}(\mathrm{H})$, $\mathrm{pl}(\mathrm{H})]$ where the $\operatorname{bel}(\mathrm{H}) \leq \mathrm{P}(\mathrm{H}) \leq \mathrm{pl}(\mathrm{H})$. Also, the
Dempsrter Shafer theory use to combine evidence that celled joint mass in rule of Dempsrter Shafer theory:

$$
\begin{gathered}
m_{1} \oplus m_{2}(\varnothing)=0 \\
m_{1} \oplus m_{2}(A)=\frac{1}{1-K} \sum_{B \cap C=A \neq \emptyset} m_{1}(B) m_{2}(C)
\end{gathered}
$$

Where:

$$
K=\sum_{B \cap C=\emptyset} m_{1}(B) m_{2}(C)
$$

In data fusion, the Dempsrter Shafer theory method use in decision stage to make decision according to belief in hypothesis [16].

\subsection{Evaluation of Decision Methods}

The most wildly known method in statistical identification methods is the Bayesian method, it is known for algorithm simplicity, it is used in many tracking application whither it was applied as indoor [17], or outdoor approaches such as mobile robotics [18]. However, these methods have several pitfalls of its own, such as it weakness in handling faults data inputs efficiently, it cannot distinguish if the data coming from the sensors are correctly found or it wrong due to the environmental noise or even sensor damaged, and that might cause some incorrect estimation [19]. Moreover, to overcome such a pitfall an generalization method of Bayesian is developed which called The Dempster-Shafer method, it overcome and uncertainly of Bayesian method, one of the main difference between the Dempster-Shafer method and Bayesian method is that the Dempster-Shafer method uses the combination of an events instead individual event like in Bayesian and that what make it more flexible, however in a comparative study applied in air surveillance [20] showed that both of the method had the ability to track and object correctly and the only difference is that the implementation of Bayesian method was more simpler and had more probabilities for the correct decision, while Dempster-Shafer method showed more sturdiness in noise and imprecise prior information disturbance. However, the modified method of Bayesian method showed more efficient, extensible, and theoretically acceptable method for managing uncertainty [19].

\section{CONCLUSION}

At the end, this paper reviews two of the main methods of data fusion, which are data fusion estimation and decision fusion, these methods are applied to provide meaning to the collected data, the data fusion estimation methods has two kind of filters Kalman and practical filter, these filters are applied according to the nature of the application such as Kalman filter are applied to collect linear data from simple systems, in the other hand practical filter is applied to nonlinear more complex system. in the other hand the decision methods are mathematical formulas applied to have statistical estimation, these methods discussed in this paper are Bayesian and Dempster-Shafer method, the methods are applied for simple system while the Dempster-Shafer are the generalized method of Bayesian. the Dempster-Shafer shows more accuracy in estimation for more complex system, all these methods can be combined or stand alone to calculate and estimate the data of the system, these methods are essentials in robotics and object tracking approaches. 


\section{REFERENCES}

[1] "Multisensor Fusion Of Target Attributes And Kinematics". Decision And Control Including The Symposium On Adaptive Processes. IEEE, 1981. Print.

[2] "Multisensor Integration And Fusion: Issues And Approaches". Orlando Technical Symposium. 1988. Print.

[3] Elmenreich, Wilfried. An Introduction To Sensor Fusion. Austria: N.p., 2002.

[4] Azimirad, Ehsan, And ,Javad Haddadnia. "A Comprehensive Review Of The Multi-Sensor Data Fusion Architectures". Journal of Theoretical and Applied Information Technology 71.1992-8645 (2015): n. pag. Print.

[5] Xinhan, Huang, and Wang Min. "Multi-Sensor Data Fusion Structures In Autonomous Systems". Lnlernailonal Symposium On Intelligent Control. Houston, Texas: IEEE, 2003. Print.

[6] H. Choset, K. Lynch and S. Hutchinson, Principles of robot motion, 1st ed. Cambridge, Mass.: Bradford, 2005.

[7] "How a Kalman filter works, in pictures | Bzarg", Bzarg.com, 2017. [Online]. Available: http://www.bzarg.com/p/how-a-kalman-filter-works-inpictures/. [Accessed: 19- Apr- 2017].

[8] Faragher, Ramsey. "Understanding The Basis Of The Kalman Filter Via A Simple And Intuitive Derivation". Signal Processing Magazine 2012: n. pag. Print.

[9] Salmond, David, and Neil Gordon. An Introduction To Particle Filters. 2015. Print.

[10] Castanedo, Federico. "A Review Of Data Fusion Techniques". The Scientific World Journal 2013 (2013): 1-19. Web.

[11] Lekkala, Kiran Kumar, and Vinay Kumar Mittal. "Accurate And Augmented Navigation For Quadcopter Based On Multi-Sensor Fusion". 2016 IEEE Annual India Conference (INDICON) (2016): n. pag. Web. 14 Apr. 2017.

[12] LaViola, J.J. "A Comparison Of Unscented And Extended Kalman Filtering For Estimating Quaternion
Motion". Proceedings of the 2003 American Control Conference, 2003. n. pag. Web. 14 Apr. 2017.

[13] Rampelli, M., and D. Jena. "Advantage Of Unscented Kalman Filter Over Extended Kalman Filter In Dynamic State Estimation Of Power System Network". Michael Faraday IET International Summit 2015 (2015): n. pag. Web. 14 Apr. 2017.

[14] Zeng, Chan, and Weimin Li. "Application Of Extended Kalman Filter For Tracking High Dynamic GPS Signal". 2016 IEEE International Conference on Signal and Image Processing (ICSIP) (2016): n. pag. Web. 14 Apr. 2017.

[15] Marron, M. et al. "Comparing A Kalman Filter And A Particle Filter In A Multiple Objects Tracking Application". 2007 IEEE International Symposium on Intelligent Signal Processing (2007): n. pag. Web. 14 Apr. 2017.

[16] K. Khoshelhama, C. Nardinocchia and S. Nedkova, "A Comparison Of Bayesian And Evidence-Based Fusion Methods For Automated Building Detection In Aerial Data", The International Archives of the Photogrammetry, Remote Sensing and Spatial Information Sciences, vol. 7, p. 1184, 2008.

[17] Abdulhafiz, Waleed A., and Alaa Khamis. "Bayesian Approach With Pre- And Post-Filtering To Handle Data Uncertainty And Inconsistency In Mobile Robot Local Positioning". Journal of Intelligent Systems 23.2 (2014): n. pag. Web.

[18] Abdulhafiz, Waleed A., and Alaa Khamis. "Bayesian Approach With Pre- And Post-Filtering To Handle Data Uncertainty And Inconsistency In Mobile Robot Local Positioning". Journal of Intelligent Systems 23.2 (2014): n. pag. Web.

[19] Abdulhafiz, Waleed A., and Alaa Khamis. "Handling Data Uncertainty And Inconsistency Using Multisensor Data Fusion". Advances in Artificial Intelligence 2013 (2013): 1-11. Web.

[20] Leung, H., and Jiangfeng Wu. "Bayesian And DempsterShafer Target Identification For Radar Surveillance". IEEE Transactions on Aerospace and Electronic Systems 36.2 (2000): 432-447. Web. 